\title{
Cushing's Syndrome After Treatment: Changes in Cortisol and ACTH Levels, and Amelioration of the Depressive Syndrome
}

\author{
Monica N. Starkman, David E. Schteingart, and M. Anthony Schork
}

\section{Received August 5, 1985; first revised version received April 16, 1986; second revised version} received June 6. 1986; accepted July 10, 1986.

\begin{abstract}
Twenty-three patients with pituitary adrenocorticotropic hormone (ACTH)-dependent Cushing's syndrome were studied before and after treatment. The relationship between the amelioration of the depressive syndrome and changes in cortisol and ACTH levels was investigated. There was a significant difference in mean change in 24-hour urinary free cortisol (UFC) excretion for changes in the depressed mood score from first to last visit. There were also significant correlations between decreases in UFC and decreases in both the depressed mood score and the modified Hamilton depression score. These relationships were not found for ACTH. Furthermore, with cortisol decreased to normal levels, continued high ACTH levels did not prevent improvement in depressed mood. The possibility that cortisol may also play a role in the pathogenesis and/or maintenance of the mood disorder in psychiatric patients is discussed.
\end{abstract}

Key Words. Cushing's syndrome, depressed mood, symptom remission, cortisol, adrenocorticotropic hormone.

Assessment of the hypothalamic-pituitary-adrenal axis has become important in psychiatry. The dexamethasone suppression test (DST) is a major investigative tool in the evaluation of patients with primary affective disorder (Carroll ct al., 1981). In addition, patients with a wide variety of psychiatric diagnoses who are DST nonsuppressors have been found to be more symptomatic on admission (e.g., anxiety, sleep disorder, difficulty with attention, and low energy) than patients who are DST suppressors (Reus, 1982).

Cushing's syndrome is, of course, the classic disease in which hypersecretion of cortisol and diminished suppression of cortisol by dexamethasone are pathognomonic features. Emotional disturbance is also present in Cushing's syndrome, and has been recognized as a feature of the disease since Harvey Cushing's original description in 1932. In a study of 35 unselected consecutive patients with Cushing's syndrome, we

\footnotetext{
The research reported was presented, in part, at the Annual Meeting of the American Psychosomatic Society, New York, NY, March 25, 1983.

Monica N. Starkman, M.D., M.S., is Director, Adult Service Consultation-Liaison Program, and Associate Professor, Department of Psychiatry; David E. Schteingart, M.D., is Professor, Department of Internal Medicine; M. Anthony Schork, Ph.D., is Professor, Department of Biostatistics, School of Public Health, University of Michigan. (Reprint requests to Dr. M.N. Starkman, Adult Psychiatric Hospital, Box 0705, University Medical Center, 1500 E. Medical Center Dr., Ann Arbor, MI 48109-0705, USA.)
}

0165-1781/86/\$03.50 (c) 1986 Elsevier Science Publishers B.V. 
described a constellation of psychiatric symptoms including impairment in affect (depressed mood and crying), cognitive functions (decreased concentration and memory), and vegetative functions (decreased libido and insomnia) (Starkman et al., 1981). Additional symptoms of greater severity such as paranoid and confusional states were much less frequent and were associated with particularly high levels of cortisol and adrenocorticotropic hormone (ACTH) (Starkman and Schteingart, 1981). The psychiatric symptoms that develop in Cushing's syndrome and the quality of the symptoms argue for a pathogenesis over and above a nonspecific response to severe physical illness. Irritability and decreased libido occur early, often before patients are aware that they have any physical problems other than a steady increase in weight. Depressed mood is experienced not simply as the demoralization common to patients with medical illness, but also as episodic sadness and crying often occurring in the absence of depressive thought content. In addition, the characteristic disorders of memory and concentration occur in patients who have no disorientation or overt clouding of consciousness.

We now report our findings in 23 patients with spontaneous pituitary ACTHdependent (PAD) Cushing's syndrome studied longitudinally from time of diagnosis through treatment. Our results indicate that improvement in the depressive syndrome is associated with treatment that effectively lowers levels of cortisol, despite continued high levels of ACTH.

\section{Methods}

Patients. Twenty-three patients with PAD Cushing's syndrome studied psychiatrically before medical treatment were available for reevaluation after treatment was initiated. Three patients were black; the remainder, white. Of these patients, 5 were male and 18 were female, approximating the ratio of male to female usually seen in this disorder. The age range was 19-60 years, with a mean of 36.9 years and a median of 35 years.

The initial diagnosis of Cushing's syndrome was established by clinical criteria plus the demonstration of excessive cortisol secretion as measured by abnormalities in urinary free cortisol, cortisol secretion rate, and plasma cortisol; absence of normal circadian rhythm of cortisol; and failure to suppress normally after $2 \mathrm{mg}$ of dexamethasone. While in a few individual cases one or another hormone level fell within the normal range, the other parameters listed above made the diagnosis clear. Before treatment, the etiological type of Cushing's syndrome had been determined for each patient on the basis of baseline cortisol and ACTH levels, as well as the ACTH response to dexamethasone suppression and metyrapone stimulation. The 23 patients with PAD Cushing's syndrome did not have a radiographically detectable pituitary tumor.

The patients received treatment with mitotane, a drug that acts by blocking steroid biosynthesis directly at the level of the adrenal gland (Schteingart et al., 1980). In addition, the patients received pituitary irradiation, a treatment that was expected, after several years, to produce an eventual decline in ACTH production. Onc patient was treated with transphenoidal pituitary surgery only.

Patients were evaluated at variable intervals once treatment began. Since patients entered the study in a consecutive fashion, some had been followed for longer periods of time, some for shorter periods. The number of months elapsed between the first and last recorded visit was therefore variable, ranging between 2 and 72 months, with a mean and a median each of 24 months.

Psychiatric Evaluation. The details of the semistructured psychiatric interview have been described previously (Starkman et al., 1981). Forty-five items covering affective, vegetative, 
and cognitive disturbances were studied by symptom report and/or observation during mental status examination. Items studied by symptom report were scored on the basis of the patient's experience of the symptom in the 4 weeks before the evaluation, as described to the examiner (MNS). Although the Research Diagnostic Criteria (RDC) are prohibited for use with such patients because of the existence of a specific organic disease causing both physical and mental symptoms, many items from the RDC were incorporated into the structured portion of the interview. For the followup visits, particular attention was given to the change in each item studied, such as depressed mood, with regard to the patient's description of alteration in quality, intensity, and frequency. Each symptom was scored separately on the same 4-point rating scale (absent, mild, moderate, or severe) established for the initial evaluation. Symptoms were further characterized as having improved, worsened, or remained the same compared to the prior visit.

We also used the Hamilton Rating Scale for Depression, a composite of the affective, vegetative, and cognitive items which constitute the depressive syndrome. We modified the standard Hamilton score (Hamilton, 1967) by omitting items 12,15, 16, and 17, which are either not appropriate in patients with Cushing's syndrome (hypochondriasis, loss of insight) or involve gastrointestinal symptoms and weight loss that could be affected by the pharmacotherapy with mitotane. Thus, these scores are useful for the longitudinal comparisons that are the focus of this article, but should not be taken as equivalent to standard Hamilton depression scores.

The depressed mood score, derived from the patient's self-report of degree of depressed mood, and the modified Hamilton score were usually (although not always) of the same relative magnitude. Spearman's rank-order correlation did show a statistically significant correlation between the depressed mood score and the modified Hamilton depression score $(\rho=0 . \%$, $p<0.01$ ).

At both the initial and followup psychiatric evaluations, the evaluator (M.N.S.) was unaware of the hormone levels of the patient.

Hormone Analysis. Plasma samples for ACTH were obtained several times during the day for assessment of circadian rhythm. Values were found to vary little during the day, a characteristic of Cushing's syndrome, both at initial and followup evaluations. Although efforts were made to obtain the entire set of hormone levels for each patient at each visit, in several instances, a particular hormone level was not available because of technical difficulties (usually the $10 \mathrm{p} . \mathrm{m}$. sample). Since the 8 a.m. samples were available most consistently, these values were used for the statistical analysis of plasma ACTH levels.

Twenty-four-hour urinary free cortisol excretion, an integrative measure of cortisol, was measured by radioimmunoassay (Dash et al., 1975).

Plasma ACTH was measured by radioimmunoassay, using the method of Vague et al. (1971). The antiserum for this assay was generously provided to us by Vague, is used at a dilution of 1:800,000, has a low cross-reactivity with other peptides, and is capable of detecting ACTH in plasma in concentrations as low as $6 \mathrm{pg} / \mathrm{ml}$. Separation of free from bound hormone is carried out with $1.5 \%$ charcoal suspension. The trace is prepared using extracted human ACTH 1-39 provided by C.H. Li and iodinated with $125 \mathrm{I}$ by the chloramine T method of Hunter and Greenwood (1962). Each sample is run in four dilutions. Nonparallel samples are reassayed and, if necessary, extracted and purified by Sephadex G50 column fractionation before radioimmunoassay. Quality control studies are carried out with each assay using standard sera and carryover samples from previous assays. The coefficient of variation is $4.2 \% \pm 1.2 \%$ for $20 \%$ binding, and $8.4 \%+2.4 \%$ for $80 \%$ binding. The same antiserum and assay method was used for all samples.

Statistical Analysis. The raw values of hormone levels in plasma and urine were not normally distributed. Log transformations gave distributions with better fit to normal distributions and were, therefore, used for the analyses. A one-way analysis of variance was used to ascertain whether there were statistically significant differences in mean changes in 24-hour urinary free cortisol and in ACTH for changes in the depressed mood symptom score from first to last visit (categorized as improved, no change, or worse). Analysis of covariance with time elapsed from 
first to last visit as a covariate was also used. For this analysis, change in depressed mood score was categorized by the magnitude of the change in point score from first to last visit $(0,+1,+2$, +3 ). Multiple regression analyses using the modified Hamilton depression scores (categorized by magnitude of change in point score from first to last visit: -4 to $-1,0$ to 2,3 to 9,10 to 14) as the dependent variable and urinary free cortisol and $\mathrm{ACTH}$ as the independent variables were computed.

Pearson's product-moment correlation coefficients were used to study the relationship of changes in modified Hamilton depression scores from first to last visit with changes in cortisol and ACTH levels. Nonparametric Spearman rank-order correlations were used to study the relationship of changes from first to last visit in symptoms such as depressed mood, which were scored on a 4-point ordinal scale, with changes in cortisol and ACTH levels. Results are reported as mean $\pm \mathrm{SD}$.

\section{Results}

Remission of Hypercortisolemia. Twenty-four-hour urinary free cortisol (UFC) is used as an integrative measure of cortisol excretion. By 3 months after the initiation of treatment, 9 of $23(39 \%)$ patients had normal UFC excretions. By 6 months, 18 of 23 $(70 \%)$ had normal UFC excretions. The mean number of months from the evaluation and initiation of treatment to normalization of cortisol levels, as measured by 24 -hour UFC excretion, was 9 . The range was $1-42$ months, with a median of 5 months. At their last visit and the psychiatric evaluation reported here, 17 patients $(73 \%)$ had normal or low cortisol levels. In contrast, only eight patients $(35 \%)$ had normal or low ACTH levels. The circadian rhythm of ACTH and cortisol secretion was still absent for most patients at the last visit.

UFC and ACTH values at $8 \mathrm{a} . \mathrm{m}$. are shown individually in Table 1 . At the initial visit, mean UFC was $689.6 \pm 639$ (normal range: $30-100 \mu \mathrm{g} /$ day). At the last visit, mean UFC was $89 \pm 61.3$. This difference is statistically significant $(p<0.001)$. At the initial visit, mean ACTH was $210.3 \pm 195$ (normal range: $52-92 \mathrm{pg} / \mathrm{ml}$ ). At the last visit, mean ACTH was $214.2 \pm 315.6$. This difference is not statistically significant.

Improvement in Depressed Mood: Qualitative Description. In our initial study, we characterized the mood disturbances in untreated spontaneous Cushing's syndrome (Starkman et al., 1981). Depressed mood was present in 75\% of the patients in the sample. Many patients stated that they would wake up depressed and remain depressed throughout the day or the next day as well. Alternatively, the onset of depressed mood and/or crying might occur during the day. Patients stressed that the onset of depression could be sudden, at times with a rapid shift at some point during the day. Some patients described hypersensitivity and oversentimentality as determinants leading to crying spells. For some patients, crying was experienced as their only available behavioral response to anger, frustration, and feelings of inability to respond effectively. Patients also described the spontaneous onset of depressed mood or crying in the absence of any preceding upsetting thought or event. There was a range in the intensity of depressed mood. Some patients described short spells of sadness; others experienced feelings of hopelessness and giving up. Six patients had had suicidal thoughts, and two of these patients had made suicide attempts since the onset of the Cushing's syndrome. Social withdrawal, when present, was related to feelings of discomfort in large groups. Patients experienced shame because of their 


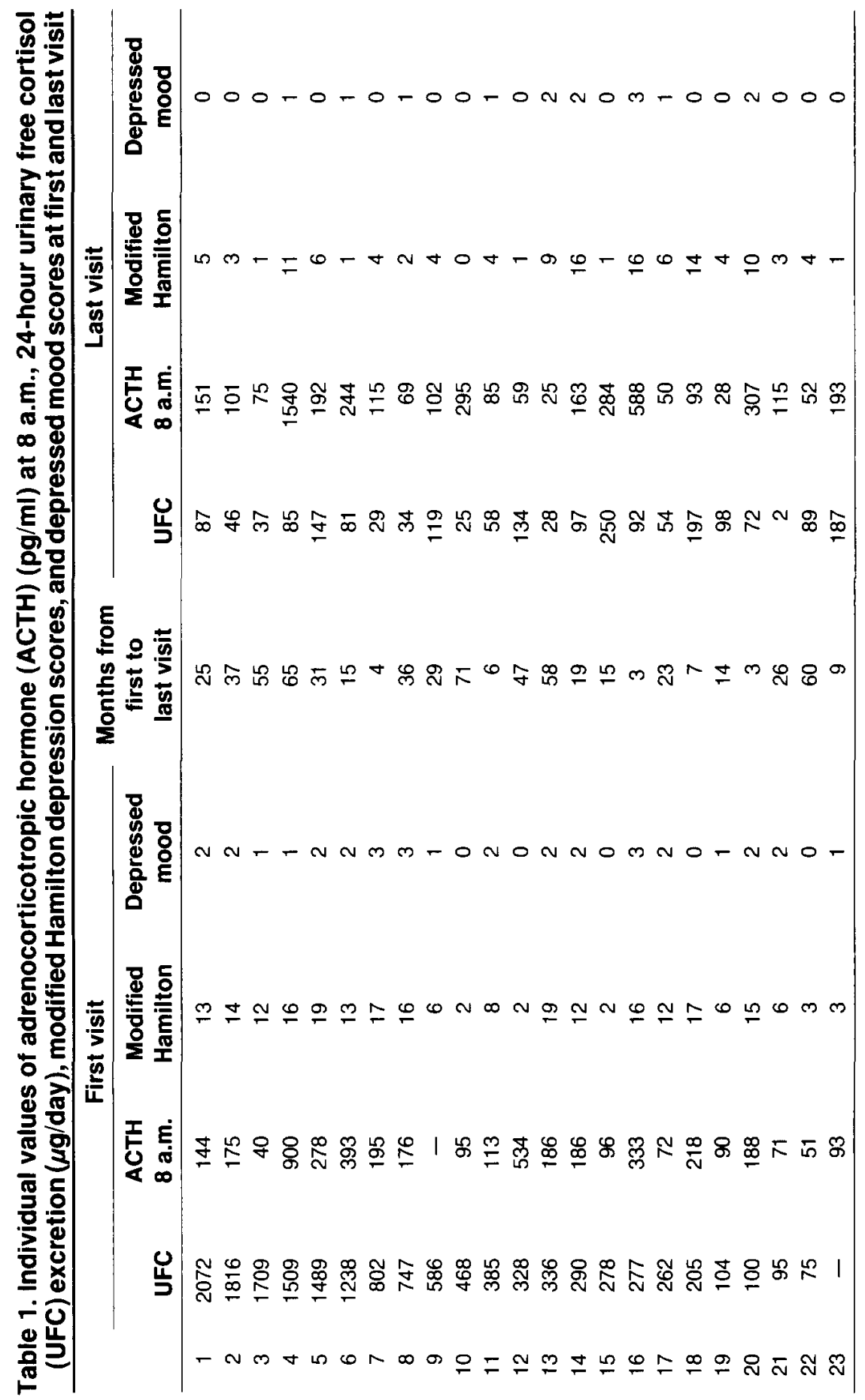


physical appearance and did not wish to be seen in public. Moreover, the unstructured setting of a large group elicited a decreased sense of focus, alertness, and clarity that led to a generalized sense of uneasiness. Most patients reported an increased desire to have contact with significant family members. As noted previously, however, sporadic withdrawal might occur because of the patient's need to remove him/herself from the situation of overstimulation that elicited the fear of impending emotional dyscontrol. Guilt, when present, was not excessive, self-accusatory, or irrational. It was related primarily to remorse about the frequent and uncontrollable angry outbursts and inability to function as well as previously at work and in the family. Hopelessness, when present, was attributed to the existence of a chronic illness with increasing physical and emotional disability that so far had proved undiagnosable and untreatable. The time course of the mood disturbances was noteworthy. Patients reported that their mood disturbances were intermittent rather than sustained. Although there were intervals when they felt lack of pleasure, these patients did not describe persistent anhedonia. They did not experience the unrelenting, unremitting inability to experience pleasure that is characteristic of patients with melancholic major depressive illness. There were intervals when they retained the capacity for pleasure and found enjoyment in hobbies, activities, and interpersonal relationships. At times, some patients found it difficult to initiate such activities; once others mobilized them, they would enjoy them. The duration of each depressive episode was usually 1 to 2 days and rarely longer than 3 days at a time. A frequent weekly total of dysphoria was reported as being 3 days per week. There was no regular cyclicity, however, so that patients could not predict when a day of depression would occur.

In the treated patients, improvements in depressed mood were characterized by a decrease in the frequency of days when the patient felt depressed. In addition, each episode would last a shorter period of time, perhaps only a few hours instead of a day or two. The patients also described a change in quality of the depressive mood, so that they no longer experienced being in a "total depression," or felt that it was "deep" or all-encompassing. They no longer became depressed without some external precipitating event. They noted that now the mood change came on gradually and disappeared gradually rather than appearing suddenly as before. Crying was less frequent, less easily elicited by environmental upsets, occurred only with some identifiable external precipitant, and was of shorter duration.

Improvement in Depressed Mood Scores and Modified Hamilton Depression Scores. At the first visit (pretreatment), 18 of 23(78\%) patients with PAD Cushing's syndrome reported depressed mood. At the last visit, 9 of these 18 patients reported normal mood, while an additional four reported improvement in depressed mood. The remaining five patients reported no improvement in depressed mood, although in three of the five the modified Hamilton score had improved by $30 \%$ or more. The difference (decrease) in mean depressed mood score from first to last visit was statistically significant $(p<0.001)$.

Modified Hamilton depression scores and depressed mood scores at the first and last visits are given for each patient in Table 1 . At first visit, 14 of 23 patients had modified Hamilton scores $\geqslant 10$. At last visit, five patients had modified Hamilton scores of $\geqslant 10$. Of these five, three had been treated $<1$ year. The mean modified 
Hamilton depression score was $10.8 \pm 5.8$ at the initial visit, and $5.5 \pm 4.8$ at the last visit. The difference (decrease) in mean modified Hamilton depression score from first to last visit was statistically significant $(p<0.001)$.

\section{Relationship of Depressed Mood Scores and Modified Hamilton Depression} Scores to Changes in Hormone Levels. For the first visit (pretreatment), an analysis of variance revealed no significant differences in mean UFC or mean ACTH levels for the four depressed mood intensity categories (absent, mild, moderate, or severe). For individuals with UFC levels $\geqslant 1000 \mu \mathrm{g} /$ day, mean UFC was $1639 \mu \mathrm{g} / \mathrm{day}$, and mean modified Hamilton was 14.5. For individuals with UFC levels between 100 and $1000 \mu \mathrm{g} / \mathrm{day}$, mean UFC was $369 \mu \mathrm{g} /$ day and mean modified Hamilton was 10.7 . For individuals with UFC levels $<100 \mu \mathrm{g} /$ day, mean UFC was $86 \mu \mathrm{g} /$ day, and mean modified Hamilton was 4 . The modified Hamilton depression scores at the first pretreatment visit showed a weak correlation with UFC $(r=0.39, p=0.08)$. Inspection of Table 1 indicates that at the higher levels of 24-hour UFC, higher modified Hamilton scores occurred consistently, but at the lower levels of 24-hour UFC elevation, modified Hamilton scores could be either high or low.

Several statistical tests were used to study the relationship of changes in the depressed mood score and in the modified Hamilton score with the changes in hormone levels that were brought about by treatment. ANOVA revealed that there was a statistically significant difference in mean change in UFC excretion for changes in depressed mood score from first to last visit $(F-5, d f-2 / 18, p<0.05)$. To study whether these differences remain significant after adjustment for the length of time patients were in the study, an analysis of covariance, using time in months elapsed from first to last visit as a covariate, was done. There was a significant effect of time. Nevertheless, the analysis revealed that covarying on time did not reduce the significance of the differences in mean UFC levels for the different mood score changes.

Correlational analyses were also completed. Spearman's rank-order correlation showed a statistically significant correlation between change (decrease) in UFC from first to last visit and change (decrease) in the depressed mood score $(\rho=0.67, p<$ 0.01 ). Pearson's product-moment correlation showed a statistically significant correlation between change (decrease) in the 24-hour UFC excretion from first to last visit and change (decrease) in the modified Hamilton depression score $(r=0.66$, $p<0.001$ ). Similarly, there was a statistically significant correlation of change (decrease) in UFC excretion measured as \% change and change (decrease) in the modified Hamilton depression score $(r=0.57, p<0.01)$.

In contrast to these findings with cortisol, analyses of variance for ACTH revealed no significant differences in either mean absolute or \% change in ACTH for change in depressed mood scores from first to last visit. In addition, there was no significant correlation between changes in ACTH level and change in the depressed mood scores or the modified Hamilton depression scores from first to last visit.

Conjoint Relationship of Cortisol and ACTH and Depressed Mood. Multiple least-squares regression analysis of the change in the modified Hamilton score on the change in both UFC and ACTH was statistically significant $(F=9.4, d f=2 / 18, p<$ 
0.01 ), with the change in UFC being the only significant contributor $(t=4.1, p<$ 0.001 ).

To explore further the conjoint relationship of cortisol and ACTH to depressed mood, the improvement in depressed mood score and modified Hamilton depression score in patients whose cortisol and ACTH levels had both normalized by the last visit was compared with that of patients in whom only cortisol levels were normalized while ACTH levels remained elevated. Of the six patients with depressed mood at first visit who subsequently demonstrated both normal UFC and ACTH levels at the last visit, five of six showed improvement in the depressed mood score. Of the nine patients with depressed mood at first visit who showed normal UFC levels but continued to have elevated ACTH levels at the last visit, six showed improvement in depressed mood scores despite the continuing elevation of ACTH, while three showed no improvement.

Six of six patients who achieved both normal UFC and normal ACTH levels showed a decrease of $\geqslant 30 \%$ in the modified Hamilton score. Of the nine patients who achieved normal UFC but continued to have elevated ACTH levels, eight showed a decrease of $\geqslant 30 \%$ in the modified Hamilton score.

While the numbers are small, it is apparent that with cortisol decreased to normal levels, continuing high ACTH levels did not prevent improvement in depressed mood and in the depressive syndrome as measured by the modified Hamilton score.

Improvement in Other Neuropsychiatric Symptoms. Change in neuropsychiatric symptoms was studied by examining the percent of patients affected with a particular symptom at the initial visit who showed improvement in that symptom at the last visit, as measured by a decrease in the rating score for that symptom.

There was a definite improvement in psychiatric symptoms with treatment. This improvement was not uniform, however, and there was variability among symptoms in the percent of affected individuals who showed improvement with treatment. In comparison to the $72 \%(13 / 18)$ of affected patients reporting improvement in depressed mood from first to last visit, $80 \%$ of affected individuals reported improvement in concentration, $78 \%$ in late insomnia, $70 \%$ in memory, $68 \%$ in irritability, $62 \%$ in middle insomnia, and $28 \%$ in libido.

Relationship of Improvement in Other Neuropsychiatric Symptoms to Changes in Hormone Levels. Statistical tests were used to study the relationship of changes in other neuropsychiatric symptoms with changes in hormone levels brought about by treatment. For those patients demonstrating the particular symptom at the first visit, ANOVAs revealed that there were statistically significant differences in mean changes of 24-hour UFC excretion for changes in scores from first to last visit (categorized as improved, no change, or worse) for: energy level (increased) and UFC excretion (decreased) ( $F=7.7, d f=2 / 19, p<0.05$ ); libido (increased) and UFC excretion (decreased) $(F=6.3, d f=2 / 10, p<0.05)$; concentration (increased) and UFC excretion (decreased) ( $F=3.6, d f=2 / 17, p<0.05$ ). There were no significant differences in mean changes of ACTH levels for changes in any of the above mentioned symptoms. For late insomnia, there were statistically significant differences in mean changes (decreases) in both UFC $(F=3.8, d f=2 / 15, p<0.05)$ and $\operatorname{ACTH}(F=8.3, d f=2 / 15, p<0.01)$ for changes in score from first to last visit. 
Correlational analyses were also performed. There were statistically significant correlations for change (improvement) in energy and change (decrease) in UFC $(\rho=0.48, p<0.01)$; change in middle insomnia and change in UFC $(\rho=0.77$, $p<0.001)$, and change in concentration and change in $\operatorname{UFC}(\rho=0.50, p<0.05)$. Changes in ACTH showed no significant correlations with changes in any of the symptoms studied.

\section{Discussion}

In our previous study of patients with untreated Cushing's syndrome, the data suggested that when cortisol levels were elevated before treatment, low or normal ACTH levels were associated with mild rather than pronounced depressed mood, while elevated ACTH levels were equally likely to be associated with mild or pronounced depressed mood (Starkman et al., 1981). In the current study, we were able to achieve some pharmacological dissection of the relationship of ACTH and cortisol levels and depressed mood. The patients were treated with mitotane, an adrenolytic drug which promptly blocks steroid synthesis directly at the level of the adrenal gland, decreasing cortisol levels while ACTH levels do not change or may even rise above the pretreatment levels in patients with pituitary ACTH-dependent Cushing's syndrome. Concomitant pituitary irradiation then often produces an eventual decline in ACTH production but only after a period of several years.

At the pretreatment visit, there was a trend toward a weak correlation of the modified Hamilton depression scores and UFC. However, using the four-point rating scale for depressed mood score, we did not find a significant relationship between the depressed mood score and UFC. It cannot be determined whether the lack of a significant relationship is due to insufficient variability in depressed mood at the first visit, insensitivity of the four-point rating scale used, or the possibility that once a threshold of cortisol elevation is reached, the absolute level of cortisol is not related to the degree of depressed mood. These results are consistent with those in patients with primary affective disorder. Using a modified Bunney-Hamburg Scale, Rubinow et al. (1984) found that only in a minority of such patients was a correlation seen when plasma cortisol values were examined just in the depressed state, and the significant correlation was highly dependent on values in both the depressed and improved state.

Our study suggests an association of improvement in depressed mood and decrease in cortisol from pretreatment values. Kelly et al. (1983), in a series of 26 patients with Cushing's syndrome, found significant improvement in depression as measured by improvement in the Hamilton Rating Scale for Depression after treatment produced decreases in cortisol levels, although these investigators could not demonstrate a significant correlation between changes in UFC and depression ratings either at 3 or 12 months. In the studies reported here, our analysis of covariance revealed a significant effect of time, complementing the impression from previous case reports of patients with adrenal adenomas that there may be a delay of months to years from the correction of elevated cortisol levels to complete remission of psychiatric symptoms (Haskett, 1985).

Further, the results of our study indicate that with normal cortisol levels achieved by treatment, depressed mood and the depressive syndrome can improve despite the 
continuation of elevated ACTH levels. This observation is supported by two recent studies reporting improvement of depression in patients with pituitary-dependent Cushing's disease, many of whom were treated with bilateral adrenalectomy or metyrapone, an 11- $\beta$-hydroxylase inhibitor that blocks cortisol biosynthesis by the adrenal gland (Jeffcoate et al., 1979; Cohen, 1980). While ACTH was not measured in these two studies, treatment with either metyrapone or bilateral adrenalectomy does characteristically result in further increases in the already high plasma ACTH levels despite adequate corticosteroid replacement where necessary.

The present study lends support to the hypothesis that the behavioral changes of the depressive syndrome seen in Cushing's syndrome may be at least partly related to the effect of elevated levels of cortisol. It is unknown whether this reflects a direct effect on the cells of the central nervous system, on neurotransmitters, or on intracellular electrolytes due to increased sensitivity of the brain to elevated levels of $\beta$-endorphin, $\mathrm{ACTH}$, or corticotropin-releasing factor (CRF), or some other mechanism.

Evidence is accumulating that corticosteroids affect a wide variety of enzymes important in neurochemistry, and may alter mood-regulating neurotransmitter systems within the brain. In animals, hydrocortisone increased liver tryptophan pyrolase with a subsequent decrease in brain tryptophan and serotonin (Green and Curzon, 1968). In tissue culture, hydrocortisone and dexamethasone stimulated tyrosine hydroxylase, the rate-limiting enzyme in catecholamine synthesis, and inhibited choline acetyltransferase, markedly inhibiting the synthesis of acetylcholine (Schubert et al., 1980). In rat brain, corticosteroids altered the sensitivity to noradrenalin of the noradrenalin receptor-coupled adenylate cyclase system (Mobley and Sulser, 1980). Anatomical evidence for the role of cortisol in regulating mood comes from the observation that in rat brain, the highest concentration of binding sites for ${ }^{3} \mathrm{H}$-corticosterone is found not in the hypothalamus but in the limbic system: hippocampus, amygdala, and septum (McEwen et al., 1975).

Studies in patients with primary psychiatric disorders also indicate that increased cortisol levels are associated with both behavioral and physiological abnormalities. Psychiatric inpatients with high 8 a.m. values of plasma cortisol, regardless of whether they had primary endogenous depression or some other psychiatric diagnosis, were more symptomatic on admission, and on discharge had more symptoms of sleep disturbance and decreased ability to think than patients with normal values of plasma cortisol (Reus, 1982). Several investigators have reported extremely high UFC levels in subgroups of depressed patients who subsequently attempted suicide (Bunney et al., 1969; Ostroff et al., 1982). Gerner and Wilkins (1983) have recently confirmed previous observations that cerebrospinal fluid (CSF) cortisol levels are elevated in depressed patients as compared to normals, and that there is a correlation of CSF cortisol and the severity of depression. As regards physiological effects, evidence for the existence of somatic effects of glucocorticoid excess in depressed DST nonsuppressors, as measured by discriminant function analysis of hematological cellular and blood chemistry findings, has been reported (Reus and Miner, 1985). A decreased sensitivity of lymphocytes to glucocorticoid-induced immunosuppression has been found in depressed patients as well, possibly due to down-regulation of the lymphocyte glucocorticoid receptor (Lowy et al., 1984). 
In summary, we studied 23 patients with PAD Cushing's syndrome before and after treatment. Improvement in depressed mood was significantly associated with lowering of the elevated cortisol levels. With cortisol decreased by treatment to normal levels, continued high ACTH levels did not prevent improvement in depressed mood. The mechanism underlying the apparent effect of cortisol on the depressed mood in Cushing's syndrome is unknown. In psychiatric patients with primary affective disease, behavioral and physiologic abnormalities have also been shown to be associated with increased cortisol levels. Thus, cortisol may play a role in the pathogenesis and/or maintenance of the mood disorder in psychiatric patients as well.

Acknowledgment. This research was supported in part by U.S. Public Health Service Grant 5 Mol RR 4216.

\section{References}

Bunney, W.E., Jr., Fawcett, J.A., Davis, J.M., and Gifford, S. Further evaluation of urinary 17-hyd roxy-corticosteroids in suicidal patients. Archives of General Psychiatry, 21, 138(1969).

Carroll, B.J., Feinberg, M., Greden, J.F., Tarika, J., Albala, A.A., Haskett, R.F., James, N. Mcl., Kronfol, Z., Lohr, N., Steiner, M., de Vigne, J.P., and Young, E. A specific laboratory test for the diagnosis of melancholia: Standardization, validation, and clinical utility. Archives of Gencral Psychiatry, 38, 15 (1981).

Cohen, S.I. Cushing's syndrome: A psychiatric study of 29 patients. British Journal of Psychiatry, 136, 120 (1980).

Cushing, H. The basophil adenomas of the pituitary body and their clinical manifestations (pituitary basophilism). Bulletin of the Johns Hopkins Hospital, 50, 137 (1932).

Dash, A.J., England, B.G., Midgley, A.R., and Niswender, G.D. Specific non-chromatographic radioimmunoassay for human plasma cortisol. Steroids, 26, 647 (1975).

Gerner, R.H., and Wilkins, J.N. CSF cortisol in patients with depression, mania or anorexia nervosa and in normal subjects. American Journal of Psychiatry, 140, 92 (1983).

Green, A.R., and Curzon, G. Decrease of 5-hydroxytryptamine in the brain provoked by hydrocortisone and its prevention by allopurinol. Nature, 220, 1095 (1968).

Hamilton, M. Development of a rating scale for primary depressive illness. British Journal of Social and Clinical Psychology, 6, 278 (1967).

Haskett, R.F. Diagnostic categorization of psychiatric disturhance in Cushing's syndrome. American Journal of Psychiatry, 142, 911 (1985).

Hunter, W.M., and Greenwood, F.C. Preparation of iodine-131 labelled human growth hormone of high specific activity. Nature, 194, 495 (1962).

Jeffcoate, W.J., Silverstone, J.J., Edwards, C.R., and Besser, G.M. Psychiatric manifestations of Cushing's syndrome: Response to lowering of plasma cortisol. Quarterly Journal of Medicine, XLVIII, 465 (1979).

Kelly, W.F., Checkley, S.A., Bender, D.A., and Mashiter, K. Cushing's syndrome and depression-A prospective study of 26 patients. British Journal of Psychiatry, 142, 16 (1983).

Lowy, M.T., Reder, A.T., Antel, J.P., and Meltzer, H.Y. Glucocorticoid resistance in depression: The dexamethasone suppression test and lymphocytic sensitivity to dexamethasone. American Journal of Psychiatry, 141, 1365 (1984).

McEwen, B.S., Gerlach, J.L., and Micco, D.J., Jr. Putative glucocorticoid receptors in hippocampus and other regions of the rat brain. In: Isaacson, R., and Pribram, K., eds. The Hippocampus: A Comprehensive Treatise. Plenum Press, New York, p. 285 (1975).

Mobley, P.L., and Sulser, F. Adrenal corticoids regulate sensitivity of noradrenaline receptor-coupled adenylate cyclase in brain. Nature, 286, 608 (1980).

Ostroff, R., Giller, E., Bonese, K., Ebersole, E., Harkness, L., and Mason, J. Neuroendocrine risk factors of suicidal behavior. American Journal of Psychiatry, 139, 1323 (1982). 
Reus, V.I. Pituitary-adrenal dysinhibition as the independent variable in the assessment of behavioral symptoms. Biological Psychiatry, 17, 317 (1982).

Reus, V.I., and Miner, C. Evidence for physiologic effects of hypercortisolemia in psychiatric patients. Psychiatry Research, 14, 47 (1985).

Rubinow, D.R., Post, R.M., Gold, P.W., Ballenger, J.C., and Wolff, E.A. The relationship between cortisol and clinical phenomenology of affective illness. In: Post, R.M., and Ballenger, J.C., eds. Neurobiology of Mood Disorders. Williams \& Wilkins Company, Baltimore (1984).

Schteingart, D.E., Tsao, H.S., Taylor, C.I., Mekenzie, A., Victoria, A., and Therrien, B.A. Sustained remission of Cushing's disease with mitotane and pituitary irradiation. Annals of Internal Medicine, 92, 613 (1980).

Schubert, D., LaCorbiere, M., Klier, F.G., and Steinbach, J.H. The modulation of neurotransmitter synthesis by steroid hormones and insulin. Brain Research, 190, 67 (1980).

Starkman, M.N., and Schteingart, D.E. Neuropsychiatric manifestations of patients with Cushing's syndrome. Archives of Internal Medicine, 141, 215 (1981).

Starkman, M.N., Schteingart, D.E., and Schork, M.A. Depressed mood and other psychiatric manifestations of Cushing's syndrome: Relationship to hormone levels. Psychosomatic Medicine, 43, 3 (1981).

Vague, P.H., Oliver, C., Jacquet, P., and Vague, J. Le dosage radio-immunologique de I'ACTH plasmatique. European Journal of Clinical and Biological Research, 16, 485 (1971). 